\title{
Design and Development of Android based Multimedia Player
}

\author{
Pooja Batra Nagpal, Akshit Sharma
}

\begin{abstract}
In today`s world, many users like to use media player in order to solve the complex functions and large memory requirement in a smart phone. With a rapid growth of network and technology, multimedia technology is used in media players. As the Android based devices are getting more popular among the people, an Android based application attracts more attention. Based on Android technology and java language the design and coding of a simple, convenient, less memory requirement as well as user friendly multimedia player is shown in this paper. The player is designed in such a way to obtain higher performance; simple operations and run on the Android based mobile devices. The papers discuss about the study of multimedia player with the help of already existing media players which are available openly in the Android market and proposed system for multimedia player that will be providing the uninterrupted enjoyment for the users.
\end{abstract}

KEYWORDS - Android, Multimedia, Smartphone, Media player, Audio player

\section{INTRODUCTION}

As Science and technology is continuously developing day by day, the mobile phones are no longer just a communication device, but a platform which provides a lot of functioning and multimedia capabilities. Android is a mobile phone-based operating system which was launched my google in November 2007. Introducing Android in the electronic market, it has changed the traditional concept of mobile phones. Anyone can easily modify the operating system, appearance, functioning and restrict the usage according to personal preference of mobile phone which makes Android an attractive interface and user friendly too. The functioning of a mobile phone is much more powerful that the traditional ones hence become a smart phone. Playing a video or an audio on a smart phone has become a basic function for many users, but due to many limitations it has the main problem arises with the storage as only a limited amount of data can be stored on a mobile phone. So, a powerful online streaming multimedia player with a large variety of video and audio is needed. Therefore, many kinds of video and audio players are also developed.

\section{Manuscript Received May 20, 2020}

Pooja Batra Nagpal, Assistant Professor,Department of Computer Science, Amity University, Gurgaon, Haryana, India (email: poojabatra9@gmail.com)

Akshit Sharma, UG student, Department of Computer Science, Amity University, Gurgaon, Haryana, India
However, a lot of player applications are too fancy and devoted to appearance and functionality, causing a lot of resource wasting for the user`s mobile phone, such as large memory requirement and CPU. All of this causes a lot of inconvenience for the user as multiple programs running at the same time. The ultimate purpose of this research is to develop an online streaming video and audio player in a single application. Multimedia player based on Android application is popular in the market at the present. The development of android operating system gives a nice platform to the developers, which can learn the latest computer technology with learned knowledge, enrich oneself with the popular technology and enjoy the entertainment of the Android SDK[1].

\section{DEVELOPING ENVIRONMENT OF ANDROID}

The applications based on Android needs to run on Android environment. The configuration requirements and installation of Android environment are as follows [2]:

The software required for Developing Android Environment.

- Operating System: Windows XP or later, Linux, Mac.

- Software: Android SDK (Software Development Kit), ADT (Android Development Tools).

- JDK: JDK (Java Development Kit), Java Runtime Environment Virtual Machine.

- IDE Environment: ADT Eclipse3 or higher + Eclipse IDE.

Steps to install Android Development Environment [3]

- Install JDK (Java Virtual Machine) version 6 or higher.

- Install Eclipse tools.

- Install Android SDK (Software Development Kit).

- Install ADT plug-in, run Eclipse and click help > install new Software and select add.

- Input the SDK path in the location and click OK.

- The Setup is completed successfully..

$\bullet$

\section{REQUIREMENT ANALYSIS OF SYSTEM}

\section{A. Feasibility Analysis}

The section verifies that it is feasible to stream online video and audio on the device with Android system [4]. Other aspects ratios of the feasibility are as follows:

\section{B. Economic Feasibility}

Android mobile phone multimedia player is designed as the Android app development is free by the Android development [5]. In addition to it multimedia player is a basic need for the people. The information of necessary functions 
forms the consumers of the community, the functions which are needed by some people. The features which are seldom and can be easily understandable. The whole process of developing does not require any money that is its economic feasibility.

\section{Technical Feasibility}

A multimedia player which meets basic requirements, a deep study of JAVA, Eclipse Development Tools, Android Architecture, application framework, other knowledge of the technical field is required to design a multimedia player. All the programmers participating in the development and designing must have framework as a core of application and rules of the programmers. Based on the technology information from the market the resources, equipped with technical personnel skills of the technology and a keen curiosity to learn the technology is feasible [6].

\section{Social Feasibility}

The mobile phone market of this world is circulating millions of audio and videos resources of all kinds on the internet. This resource of achieving the online streaming on your phone seems ordinary but have become an indispensable part of the people in our world. This draws an attention for development of different kinds of mobile phone players. There are many players which devotes to fancy appearance, strong functions with a lot of resources wasted to the mobile phone of the user which brings a lot of inconvenience for the user as multitasking operation is needed[7]. To overcome these problems developing a multimedia player which has simple functions, common play function, less memory requirement, meets user needs and high quality of video and audio streaming, increases the optimization in performance.

\section{CHARACTERISTICS OF SOFTWARE DESIGN}

PROPOSED

\section{A. Reliability}

The software must be checked with its reliability. A software is reliable if it avoids faults in the process of running system and remedy troubles if any fault occurs[8].

\section{B. Reusability}

Look for redundancy of similar codes, and comes with new method reasonably and abstractly. Generic design is considered under attention.

\section{Simple Program}

To solve the complex code problems good programmers keeps the codes simple and clear.

\section{Understandability}

A clear and readable document along with the software structure makes software understandable. The understandability of the software requires keen insight and creativity of the designer and the skills.

\section{E. Testability}

The software with proper data set to conduct comprehensive test of system is known as testability.

\section{F. Open-Closed Principal}

The extension will be open to the code to adapt the new requirements. The module is extensible but can`t be modified. When the design is completed, categories won't be modified.

\section{MULTIMEDIA PLAYER}

Multimedia player is achieved by the Eclipse[9]. For developing android app, we need to install a plug-in for Eclipse: Android Development Tools. After installation, download Android SDK (12 or higher), install and configure SDK, then only we can develop a multimedia player. In this research a study of operating mechanism, media layer structure of android platform, customizable inter face of xml, the content provider can achieve a scanned file to the media files list. The media player class, Surface Flinger interface, File parsing. After all of this, we can develop an Androidbased mobile multimedia player. The realization of media library, video player, opening audio file, video, different screens and other functions. The System Flow Chart is shown here.

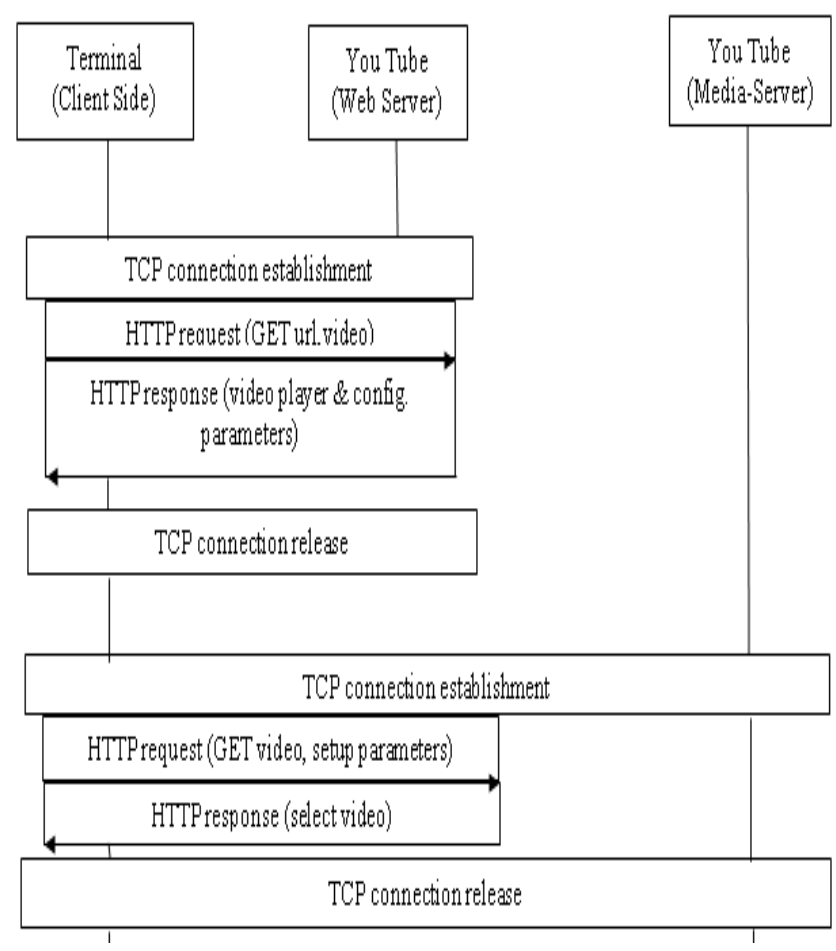

Fig 1: System Flow Chart

\section{APPLICATION FRAMEWORK}

All the API framework of the core programs is allowed to the developer. The framework of the application makes easier to reuse its components [10]. The users will be able to substitute program components by this reuse mechanism. Some other applications can release their functional components which 
allows other apps to access and use this component (Accept the security of the framework as followed[11][12]).

\section{FUNCTION DESIGN}

The interface is defined with the xml files, XML layout files control view, is not only simple, but isolate the control view logic from the java code and managed by inserted in XML files. All of the MVC principles are reflected in a better way and the principle of separation of logic and views. The software itself obtains list of media player by scanning through the online content providers across the globe. Content providers are like a bridge between data storing and searching across programs. The main functioning of the software is to achieve data sharing among online resources and searching with other apps. This is the only way to share data with other apps.

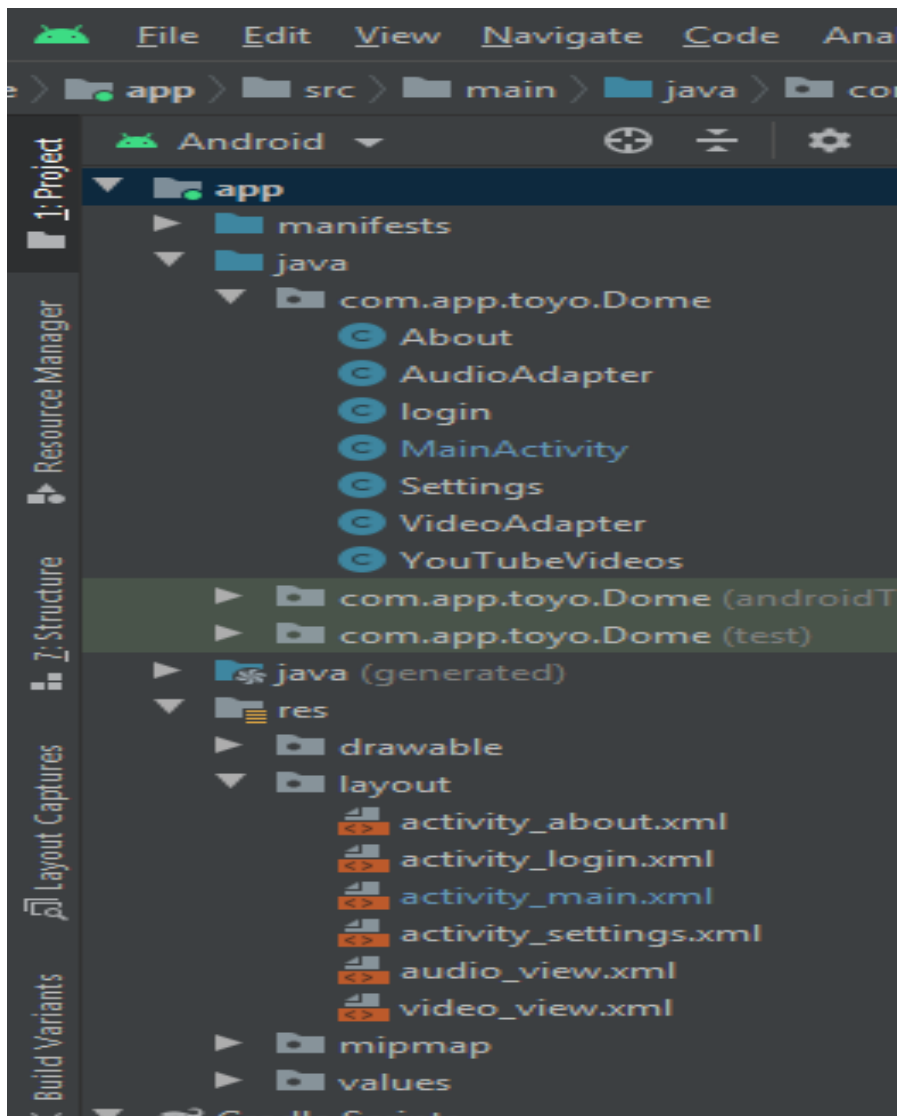

Fig 2: Function Design

\section{PLAYLIST DESIGN}

A view known as Recycler view in Android with the characteristic of Adapter attribute. The display from top to bottom is applied. The default view of the system is just a line only shown with Text View, in addition of the playlist is in user defined form. The List View shows single picture and the sufficient details in every row. We defined a class adaptive inherited based adapter. To expand the adapters by using algorithms, in which a picture and some details are shown in every line. As the Base adapter is an abstract class, we need to execute the abstract method" get View", which will return a view. View is displayed on the activity, so that the playlist interface will come out.

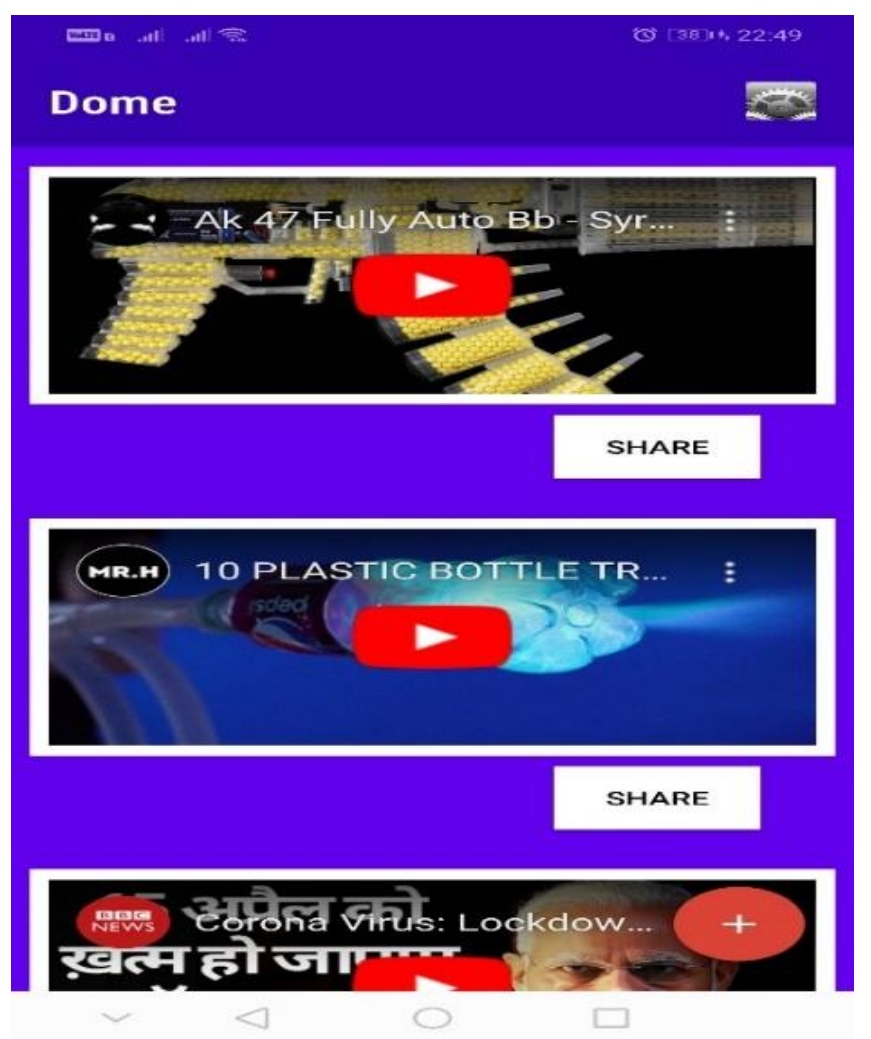

Fig 3: Video Playlist

Figure 3 shows the glimpse of video playlist while figure 4 shows the glimpse of Audio playlist.

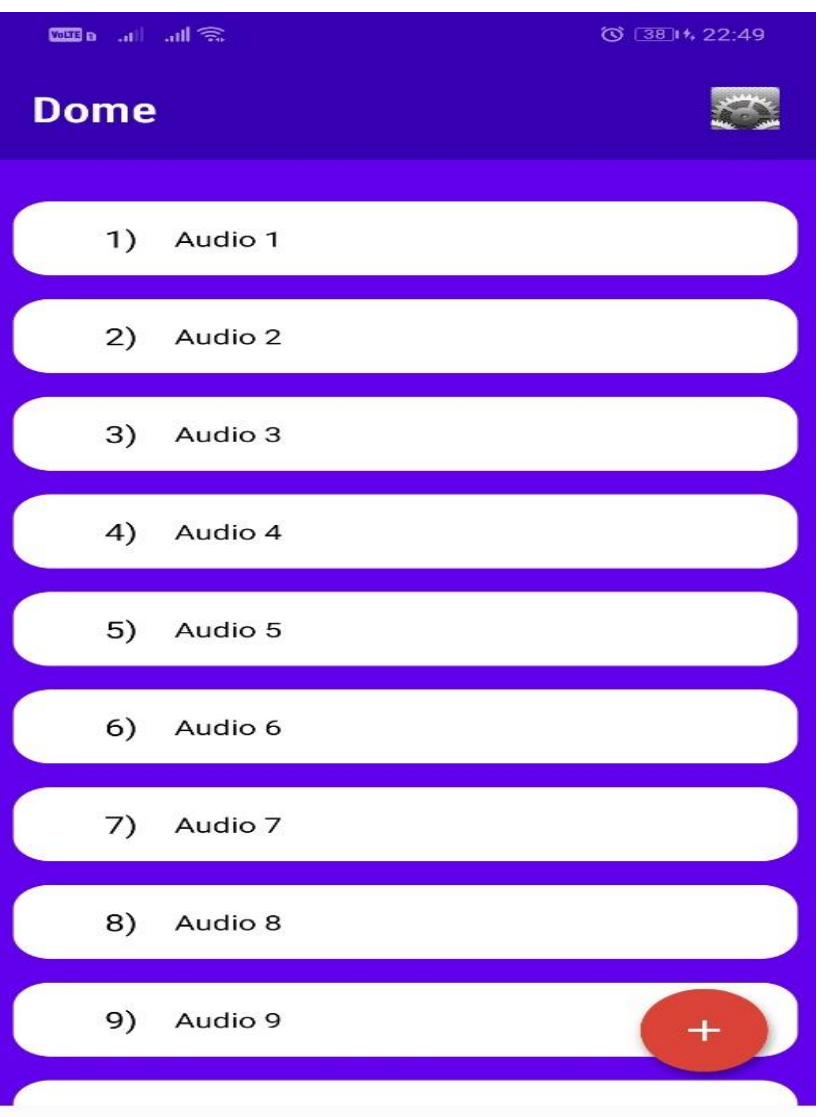

Fig 4: Audio Playlist 


\section{PLAY MODE}

A component called Radio Group which has the function of single choice at a time choice. There comes a Radio Button in the Radio Group. Multiple Radio Buttons items of the player includes one cycle at a time, the user can select only one play mode. This includes random play and play in order also. The media Player will play in a monitor which ensures that video and audio both starting and completing. When the file complete, method OnCompletionListener will be triggered. By this method the operation at the completion of the play will be processed.

\section{CONCLUSION}

By the development of the multimedia player on the Android platform, we will get a clear understanding of the overall processing of the Android system. The main part of the multimedia player is the playlists, menus, play screen, interface, security, and streaming. By all these parts, the multimedia player has achieved a preliminary scale. Based on the mentioned categories add some more features.Multimedia Player system realizes the basic functioning of the player: play, pause and stop, adjust volume by up/down, display, play mode, playlist query, and many other functions. The development has been popular in the mobile terminal development technology. The system realized multimedia player programming in the open sourced mobile platform Android.The design requires multimedia player framework, by adapting Eclipse and Java language for technical support of the system based on the Android systems. Android plug-in tools and Android SDK combine leads to the comprehensive and smooth design and development of the mobile phones.

\section{REFERENCES}

[1] M. Butler, "Android: Changing the Mobile Landscape", Pervasive Computing, (2011), pp. 4-7.

[2] B. Proffitt, "Open Android-For better and for worse", Spectrum, (2011), pp. 22- 24.

[3] K. W. Tracy, "Mobile Application Development Experiences on Apple's iOS and Android OS", Potentials, (2012), pp. $30-34$.

[4] Shabtai, Y. Fledel, U. Kanonov, Y. Elovici, S. Dolev and C. Glezer, "Google Android: A Comprehensive Security Assessment”, Security \& Privacy, (2010), pp. 35 - 44.

[5] Shabtai, Y. Fledel and Y. Elovici, "Securing AndroidPowered Mobile Devices Using SELinux", Security \& Privacy, (2010), pp. 36-44.

[6] M. Song, J. Sun, X. Fu and W. Xiong, "Design and Implementation of Media Player Based on Android", WICOM, (2010), pp. 1 - 4 .

[7] D. Gavalas and D. Economou, "Development Platforms for Mobile Applications: Status and Trends", Software, (2011), pp. 77-86.

[8] X. Zhao and D. Tian, "The Architecture Design of Streaming Media Applications for Android OS", ICSESS, (2012), pp. 280 - 283.

[9] W. Enck, M. Ongtang and P. McDaniel, "Understanding Android Security"Security \& Privacy, (2009), pp. 50 - 57.

[10] Xu, J. The Design and Implementation of Music Player Based on Android Platform, Beijing Posts and Tele communications University,2011 5:156 178 .
[11] Wang, Gai. Guide Introduction of Google Android second version, People's Posts and Telecommunications Publishing House 2009-11:263 287.

[12] Sayed Hashimi, Satya Komatineni, Dave MacLean. Master of the Android 2. 